\title{
The sum of Rademacher functions and Hausdorff dimension
}

\author{
BY TIAN-YOU HU AND KA-SING LAU \\ Department of Mathematics and Statistics, University of Pittsburgh, \\ Pittsburgh, PA 15260, U.S.A.
}

(Received 29 August 1989)

Abstract

For $0<\alpha<1$, let $f_{\alpha}(x)=\sum_{i=1}^{\infty} 2^{-\alpha i} R_{i}(x)$ for $0 \leqslant x<1$, where $\left\{R_{i}\right\}_{i=1}^{\infty}$ is the sequence of Rademacher functions. We give a class of $f_{\alpha}$ so that their graphs have Hausdorff dimension $2-\alpha$. The result is closely related to the corresponding unsolved question for the Weierstrass functions.

\section{Introduction}

For $0<\alpha<1$, let

$$
f_{\alpha}(x)=\sum_{i=1}^{\infty} 2^{-\alpha i} R_{i}(x) \quad(0 \leqslant x<1)
$$

where $R_{i}$, for $i=1,2, \ldots$, denotes the Rademacher functions : $R_{1}(x)$ is defined on $\mathbb{R}$ with period 1 , takes values 1 and -1 on the intervals $\left[0, \frac{1}{2}\right)$ and $\left[\frac{1}{2}, 1\right)$ respectively, and $R_{i}(x)=R_{1}\left(2^{i-1} x\right)$ for $i>1$. In this note we will give a sufficient condition to determine the Hausdorff dimension of the graph $\Gamma_{f_{x}}$ of $f_{\alpha}$ (denoted by $\operatorname{dim} \Gamma_{f_{x}}$ ). This is closely related to the well known open problem whether the graph of the Weierstrass function

$$
W_{\alpha}(x)=\sum_{i=1}^{\infty} \lambda^{-\alpha i} \sin \left(\lambda^{i} \pi x\right) \quad(0 \leqslant x<1),
$$

where $\lambda>1$, has Hausdorff dimension 2- $\alpha$ : see [3], p. 114 and [8]. (Note that $R_{i}(x)=\operatorname{sgn} \sin \left(2^{i} \pi x\right)$ except when $x$ is a zero of $\sin \left(2^{i} \pi x\right)$.)

Let $F_{\alpha}(y)=\left|\left\{x \in[0,1): f_{\alpha}(x)<y\right\}\right|$ be the distribution function of $f_{\alpha}$, where $|A|$ denotes the Lebesgue measure of a measurable subset $A$ of $\mathbb{R}$. Among the other results we prove

TheOREm 1-1. Suppose that $F_{\alpha}$ is absolutely continuous and $F_{\alpha}^{\prime} \in L^{p}$ for some $p>1$. Then $\operatorname{dim} \Gamma_{f_{x}}=2-\alpha$.

The function $f_{\alpha}$ can be considered as the random variable of the sum of a sequence of independent Bernoulli trials. The distribution $F_{\alpha}$ can hence be obtained as the infinite convolution of the Bernoulli distributions. It follows from a theorem of Jessen and Wintner [6] that $F_{\alpha}$ is either absolutely continuous or purely singular. The determination of which type is however, very difficult (see Erdös[2], Garsia[4], Kahane and Salem [7], Salem [9] and Wintner [10]). Although it is known that $F_{\alpha}$ is absolutely continuous for some $\alpha$ and that $F_{\alpha}$ is purely singular for some $\alpha$, a complete description for $0<\alpha<1$ is still unknown. 
Theorem $1 \cdot 1$ is proved in Section 2 ; the proof actually goes through with a weaker assumption on $F_{\alpha}$, namely, that $F_{\alpha}$ satisfies certain integrated Lipschitz condition (Theorem 2.6). The known results on the absolute continuity of $F_{\alpha}$, the relevance of the above integrated Lipschitz condition, and other remarks on the dimension of $\Gamma_{f_{z}}$ are discussed in Section 3.

\section{The theorem}

Let $\mathbb{N}=\{1,2, \ldots\}$. For $n \in \mathbb{N}$ and $0 \leqslant k \leqslant 2^{n}-1$, let $I_{n, k}$ be the dyadic intervals $\left[2^{-n} k, 2^{-n}(k+1)\right)$ in $[0,1)$, and let $\mathscr{S}$ be the class of dyadic squares in $[0,1) \times \mathbb{R}$. For $s>0$, we use $\mathscr{H}^{s}$ to denote the Hausdorff $s$-dimensional measure on $\mathbb{R}^{2}$, i.e. for any subset $E$ in $\mathbb{R}^{2}$,

$$
\mathscr{H}^{s}(E)=\lim _{\delta \rightarrow 0_{+}} \inf \left\{\sum_{i}\left(\operatorname{diam} U_{i}\right)^{s}: E \subseteq \bigcup_{i} U_{i}, \operatorname{diam} U_{i}<\delta\right\}
$$

where $\left\{U_{i}\right\}$ are open subsets in $\mathbb{R}^{2}$. The Hausdorff dimension of $E$, denoted by $\operatorname{dim} E$, is defined by

$$
\operatorname{dim} E=\inf \left\{s>0: \mathscr{H}^{s}(E)=0\right\}
$$

(which also equals $\sup \left\{s>0: \mathscr{H}^{s}(E)=\infty\right\}$ ). It is known that if we replace the $U_{i}$ by dyadic squares, the dimension of $E$ is unchanged.

In this section we will fix $0<\alpha<1$. We can hence use $f, F$ and $\Gamma$ to denote $f_{\alpha}, F_{\alpha}$ and $\Gamma_{f_{z}}$ respectively without causing confusion.

Lemma 2-1. For $0<\alpha<1, \mathscr{H}^{2-\alpha}(\Gamma)<\infty$, and hence $\operatorname{dim} \Gamma \leqslant 2-\alpha$.

Proof. The proof is the same as in [3], theorem $8 \cdot 1$ for Lipschitz functions.

Let $I_{n, k}$ be a dyadic interval in $[0,1)$, let $f$ be restricted on $I_{n, k}$ and define the corresponding distribution function $F_{n, k}$ by

$$
F_{n, k}(y)=\left|\left\{x \in I_{n, k}: f(x)<y\right\}\right| \text {. }
$$

Lemмa 2.2. (i) For $x \in I_{n, k}, f(x)=c_{n, k}+2^{-\alpha n} f\left(2^{n}\left(\alpha-2^{-n} k\right)\right)$ for some constant $c_{n, k}$, and (ii) for $y \in \mathbb{R}, F_{n, k}(y)=2^{-n} F\left(2^{\alpha n}\left(y-c_{n, k}\right)\right)$.

Proof. (i) For $x \in I_{n, k}$, using the periodicity of $R_{i}$ we can write

$$
f(x)=\sum_{i=1}^{n} 2^{-\alpha i} R_{i}(x)+2^{-\alpha n} \sum_{i=1}^{\infty} 2^{-\alpha i} R_{i}\left(2^{n}\left(x-2^{-n} k\right)\right)=c_{n, k}+2^{-\alpha n} f\left(2^{n}\left(x-2^{-n} k\right)\right) .
$$

(ii) Let $y \in \mathbb{R}$. Then by (i) we have

$$
\begin{aligned}
F_{n, k}(y) & =\left|\left\{x \in I_{n, k}: c_{n, k}+2^{-\alpha n} f\left(2^{n}\left(x-2^{-n} k\right)\right)<y\right\}\right| \\
& =\left|\left\{x \in I_{n, k}: f\left(2^{n}\left(x-2^{-n} k\right)\right)<2^{\alpha n}\left(y-c_{n, k}\right)\right\}\right| \\
& =\left|\left\{2^{-n}(u+k) \in I_{n, k}: f(u)<2^{\alpha n}\left(y-c_{n, k}\right)\right\}\right| \\
& =2^{-n}\left|\left\{u \in[0,1): f(u)<2^{\alpha n}\left(y-c_{n, k}\right)\right\}\right|=2^{-n} F\left(2^{\alpha n}\left(y-c_{n, k}\right)\right) .
\end{aligned}
$$

In the following we will give a simple proof of a special case of Theorem $1 \cdot 1$, which also serves as motivation for the more elaborate proof in Lemma $2 \cdot 5$.

Let $P: \mathbb{R}^{2} \rightarrow \mathbb{R}$ be the natural projection defined by $P(x, y)=x$. 
Theorem 2-3. If $F$ is absolutely continuous and $F^{\prime} \in L^{\infty}$, then $0<\mathscr{H}^{2-\alpha}(\Gamma)<\infty$, and hence $\operatorname{dim} \Gamma=2-\alpha$.

Proof. Let $M$ be the essential supremum of $F^{\prime}$, and let $\left\{S_{i}\right\} \subseteq \mathscr{S}$ be an arbitrary cover of $\Gamma$, where $S_{i}=I_{n_{i}, k_{i}} \times\left[y_{i}, y_{i}+2^{-n_{i}}\right)$. Then by Lemma $2 \cdot 2$ we have

It follows that

$$
\begin{aligned}
\left|P\left(S_{i} \cap \Gamma\right)\right| & =F_{n_{i}, k_{i}}\left(y_{i}+2^{-n_{i}}\right)-F_{n_{i}, k_{i}}\left(y_{i}\right) \\
& =2^{-n_{i}}\left(F\left(2^{\alpha n_{i}}\left(y_{i}-c_{n_{i}, k_{i}}+2^{-n_{i}}\right)\right)-F\left(2^{\alpha n_{i}}\left(y_{i}-c_{n_{i}, k_{i}}\right)\right)\right) \\
& \leqslant M 2^{-n_{i}(2-\alpha)} .
\end{aligned}
$$

$$
\sum_{i}\left(\operatorname{diam} S_{i}\right)^{2-\alpha}=c \sum_{i} 2^{-n_{i}(2-\alpha)} \geqslant c M^{-1} \sum_{i}\left|P\left(S_{i} \cap \Gamma\right)\right|=c M^{-1},
$$

where $c=2^{(2-\alpha) / 2}$, and hence $0<\mathscr{H}^{2-\alpha}(\Gamma)$. The assertion follows from this and Lemma $2 \cdot 1$.

The crucial step of using the boundedness of $F^{\prime}$ is inequality (1). The following two lemmas are devised to show that under appropriate hypothesis, the squares that violate (1) are relatively few.

Lemma 24. For $0<\delta<1-\alpha$, let I be a dyadic interval in $[0,1)$ of size $2^{-n}$, and let $\mathscr{S}_{1} \subseteq \mathscr{S}$ be the smallest disjoint collection of dyadic squares of size $2^{-n}$ that covers $P^{-1}(I) \cap \Gamma$. Let $\mathscr{S}_{I}^{\prime} \subseteq \mathscr{S}_{I}$ consist of those $S \in \mathscr{S}_{I}$ such that

$$
|P(S \cap \Gamma)|>|I|^{2-\alpha-\delta} .
$$

Then $\mathscr{S}_{I}^{\prime}$ has at most $\left[2^{n(1-\alpha-\delta)}\right]$ members. $([x]$ denotes the largest integer which is not greater than $x$.)

Remark. We refer to the above $S$ the 'bad' squares. Since it needs $\left[c 2^{n(1-\alpha)}\right]$ disjoint squares of size $2^{-n}$ to cover $P^{-1}(I) \cap \Gamma$, where $c$ is some constant depending only on $\alpha$, the portion of bad squares is at most $c 2^{-n \delta}$.

Proof. Let $q$ be the number of squares in $\mathscr{S}_{1}^{\prime}$. Then

and hence $q \leqslant\left[2^{n(1-\alpha-\delta)}\right]$.

$$
q|I|^{2-\alpha-\delta}<\sum_{S \in \mathscr{S}_{I}^{\prime}}|P(S \cap \Gamma)| \leqslant|I|,
$$

Lemma 2-5. Suppose that there exist $M>0$ and $\epsilon_{1}>0$ such that for each $0<\epsilon<\epsilon_{1}$, there exists $h(\epsilon)>0$ with the property that

$$
\sum_{i=1}^{q} F\left(\alpha_{i}+h\right)-F\left(\alpha_{i}\right) \leqslant M q^{\epsilon_{1}} h^{\varepsilon},
$$

for all $0<h \leqslant h(\epsilon)$, and for all finite families of disjoint intervals $\left\{\left[\alpha_{i}, \alpha_{i}+h\right)\right\}_{i-1}^{q}$. Then $\operatorname{dim} \Gamma=2-\alpha$.

Proof. Given $\delta>0$, choose $0<\epsilon<\epsilon_{1}$ close to $\epsilon_{1}$ so that $\delta \epsilon_{1}+(1-\alpha)\left(\epsilon-\epsilon_{1}\right)>0$ and denote this number by $\eta$. Let $\bar{n} \in \mathbb{N}$ be such that

$$
M \sum_{n=\tilde{n}}^{\infty} 2^{-n \eta}<\frac{1}{3}
$$

Without loss of generality, we can assume that (3) holds for all $h \leqslant 2^{(\alpha-1) \bar{n}}$. 
For any $n>\bar{n}$, let $I_{n, k}$ be a dyadic interval of $[0,1)$ and let $\mathscr{S}_{I_{n, k},}, \mathscr{S}_{I_{n, k}^{\prime}}$ be defined as in Lemma $2 \cdot 4$. Let $B_{n, k}=\bigcup_{i}\left\{S_{i}: S_{i} \in \mathscr{S}_{I_{n, k}^{\prime}}^{\prime}\right\}$ where $S_{i}=I_{n, k} \times\left[u_{i}, u_{i}+2^{-n}\right)$. Then

$$
\begin{aligned}
\left|P\left(B_{n, k} \cap \Gamma\right)\right| & =\sum_{S_{i} \in \mathscr{S}_{I_{n, k}^{\prime}}^{\prime}}\left|P\left(S_{i} \cap \Gamma\right)\right|=\sum\left(F_{n, k}\left(u_{i}+2^{-n}\right)-F_{n, k}\left(u_{i}\right)\right) \\
& =2^{-n} \sum\left(F\left(2^{\alpha n}\left(u_{i}-c_{n, k}+2^{-n}\right)\right)-F\left(2^{\alpha n}\left(u_{i}-c_{n, k}\right)\right)\right\rangle \\
& \leqslant 2^{-n} M 2^{-n(\alpha+\delta-1) \varepsilon_{1} 2^{(\alpha-1) n \varepsilon}}=M 2^{-n(1+\eta)} .
\end{aligned}
$$

Here we have used Lemmas $2 \cdot 2$ and $2 \cdot 4$ and inequality (3). Define

$$
B=\bigcup_{n \geqslant n} \bigcup_{k=0}^{2^{n}-1} B_{n, k}
$$

Then the above estimate and the inequality (4) imply that

$$
|P(B \cap \Gamma)| \leqslant M \sum_{n=\bar{n}}^{\infty} 2^{-n \eta}<\frac{1}{3} .
$$

(This means that the projection of all the bad squares of size less than $2^{-\bar{n}}$ has Lebesgue measure less than $\frac{1}{3}$.)

Now let $\mathscr{C} \subseteq \mathscr{S}$ be an arbitrary cover of $\Gamma$ such that each $S_{i} \in \mathscr{C}$ has size less than $2^{-\bar{n}}$. If $S_{i} \ddagger B$, then

$$
\left|P\left(S_{i}\right)\right|^{2-\alpha-\delta} \geqslant\left|P\left(S_{i} \cap \Gamma\right)\right|
$$

and we have

$$
\begin{aligned}
\sum_{S_{i} \in \mathcal{B}}\left(\operatorname{diam} S_{i}\right)^{2-\alpha-\delta} & =c \sum_{S_{i} \in \mathcal{B}}\left|P\left(S_{i}\right)\right|^{2-\alpha-\delta} \geqslant c \sum_{S_{i} \in \mathcal{Q}, S_{i} \ddagger B}\left|P\left(S_{i} \cap \Gamma\right\rangle\right| \\
& \geqslant c(1-|P(B \cap \Gamma)|) \geqslant \frac{2}{3} c,
\end{aligned}
$$

where $c=2^{(2-\alpha-\delta) / 2}$. Hence $\mathscr{H}^{2-\alpha-\delta}(\Gamma)>0$. This implies that $\operatorname{dim} \Gamma \geqslant 2-\alpha-\delta$. Since $\delta$ is arbitrary, we conclude that $\operatorname{dim} \Gamma \geqslant 2-\alpha$, and hence $\operatorname{dim} \Gamma=2-\alpha$ by Lemma $2 \cdot 1$.

Theorem 2.6. Suppose that there exists $p>1$ such that for every $1<\beta<p$,

$$
\lim _{h \rightarrow 0_{+}} \frac{1}{h^{\beta}} \int_{-\infty}^{\infty}|F(y+h)-F(y)|^{p} d y=0
$$

Then $\operatorname{dim} \Gamma=2-\alpha$.

Proof. We first claim that for any $h>0$,

$$
F(a+h)-F(a) \leqslant \frac{1}{h} \int_{a-h}^{a+h}(F(x+h)-F(x)) d x .
$$


Indeed,

$$
\begin{aligned}
\frac{1}{h} \int_{a-h}^{a+h}(F(x+h)-F(x)) d x= & \frac{1}{h}\left(\int_{a+h}^{a+2 h} F(x) d x-\int_{a-h}^{a} F(x) d x\right) \\
\geqslant & \frac{1}{h}\left(\left(\left.x F(x)\right|_{a+h} ^{a+2 h}-\int_{a+h}^{a+2 h}(a+2 h) d F(x)\right)\right. \\
& \left.-\left(\left.x F(x)\right|_{a-h} ^{a}-\int_{a-h}^{a}(a-h) d F(x)\right)\right) \\
= & F(a+h)-F(a) .
\end{aligned}
$$

Now for any finite family of disjoint intervals $\left\{\left[a_{i}, a_{i}+h\right)\right\}_{i=1}^{q}$, with union $U$, writing $V=\bigcup_{i=1}^{q}\left[a_{i}-h, a_{i}\right)$ we have

$$
\begin{aligned}
\sum_{i=1}^{q}\left(F\left(a_{i}+h\right)-F\left(a_{i}\right)\right) & \leqslant \frac{1}{h} \sum_{i=1}^{q} \int_{a_{i}-h}^{a_{i}+h}\left|F^{\prime}(x+h)-F(x)\right| d x \\
& =\frac{1}{h}\left(\int_{U}+\int_{V}|F(x+h)-F(x)| d x\right) \\
& \leqslant \frac{2}{h}(q h)^{1 / p^{\prime}}\left(\int_{-\infty}^{\infty}|F(x+h)-F(x)|^{p} d x\right)^{1 / p} \\
& =2 q^{1 / p^{\prime}} h^{(\beta-1) / p}\left(\frac{1}{h^{\beta}} \int_{-\infty}^{\infty}|F(x+h)-F(x)|^{p} d x\right)^{1 / p}
\end{aligned}
$$

where $1 / p+1 / p^{\prime}=1$, and $1<\beta<p$. To check that the condition in Lemma 2.5 is fulfilled, we let $M=1, \epsilon_{1}=1 / p^{\prime}, \epsilon=(\beta-1) / p$ for $1<\beta<p$, and let $h(\epsilon)$ be such that for $0<h \leqslant h(\epsilon)$,

$$
2\left(\frac{1}{h^{\beta}} \int_{-\infty}^{\infty}|F(x+h)-F(x)|^{p} d x\right)^{1 / p} \leqslant 1 .
$$

Proof of Theorem 1.1. The assertion is a corollary of Theorem $2 \cdot 6$ since $F$ is absolutely continuous and $F^{\prime} \in L^{p}$ for some $p>1$ if and only if

$$
\sup _{h \geqslant 0} \frac{1}{h^{p}} \int_{-\infty}^{\infty}|F(x+h)-F(x)|^{p} d x
$$

is bounded (see [5]), hence (5) is satisfied. It can also be proved by a direct verification of (3). Let $E=\bigcup_{i=1}^{q}\left[a_{i}, a_{i}+h\right)$. Then

$$
\begin{aligned}
\sum_{i=1}^{q}\left(F\left(a_{i}+h\right)-F\left(a_{i}\right)\right) & =\int_{E} F^{\prime}(x) d x \leqslant|E|^{1 / p^{\prime}}\left(\int_{-\infty}^{\infty}\left|F^{\prime}(x)\right|^{p} d x\right)^{1 / p} \\
& =\left(\int_{-\infty}^{\infty}\left|F^{\prime}(x)\right|^{p} d x\right)^{1 / p} q^{1 / p^{\prime}} h^{1 / p^{\prime}}
\end{aligned}
$$

where $1 / p+1 / p^{\prime}=1$. By taking $\epsilon_{1}=1 / p^{\prime}$ in Lemma 25 , condition (3) is clearly fulfilled. 


\section{Some open questions}

The Fourier transformation of the distribution function $k_{\alpha}^{\prime}$ is given by

$$
L_{\alpha}(u)=\prod_{i=1}^{\infty} \cos \left(2^{-\alpha i} u\right) \text {. }
$$

By studying the function $L_{\alpha}(u)$, Wintner [10] proved that if $\alpha=1 / n$, where $n \in \mathbb{N}$, then $F_{\alpha}$ is absolutely continuous. (Actually $F_{\alpha}^{\prime} \in L^{\infty}$ : see Garsia [4], theorem 1.8.) Erdös [2] proved that for any positive integer $m$ there exists a $\beta(m)<1$ (sufficiently close to 1) such that for almost all $\alpha$ with $\beta(m)<2^{-\alpha}<1, F_{\alpha}$ has an $m$ th derivative. On the other hand by using some algebraic number theory, Salem [9] characterized the $\alpha$ such that $L_{\alpha}(u) \nrightarrow 0$ as $u \rightarrow \infty$ as those such that $2^{\alpha}$ is a Pisot-Vijayaraghavan number. By the Riemann Lebesgue Lemma, such $F_{\alpha}$ cannot be absolutely continuous. Some more special cases including a necessary and sufficient condition for $F_{\alpha}$ to be absolutely continuous with a derivative in $L^{p}$ for some $p>1$ had also been obtained by Garsia [4]. Despite all these the following is still an open question.

Question 1. Is $F_{\alpha}$ absolutely continuous for almost all $\alpha \in(0,1)$ ?

The condition in Theorem $2 \cdot 6$ is slightly weaker than the condition that $F_{\alpha}$ is absolutely continuous with $F_{\alpha}^{\prime} \in L^{p}$ for some $p>1$.

Question 2. Does $F_{\alpha}$ satisfy (5) for all $\alpha \in(0,1)$ ?

Beyer [1] showed that the Hausdorff dimension of the level sets of $f_{\alpha}$, with $\alpha=\frac{1}{2}, \frac{1}{3}, \ldots$, is $1-\alpha$ for almost all levels. Combining this result with an argument of Marstrand [3], theorem 5.8 it can be shown that $\operatorname{dim} \Gamma_{f_{x}}=2-\alpha$ for $\alpha=\frac{1}{2}, \frac{1}{3}, \ldots$.

Question 3. Does the absolute continuity of $F_{\alpha}$ imply that the level sets have dimension $1-\alpha$ ?

Question 4. Can the argument used in this note be applied to solve the dimension problem for the Weierstrass functions $W_{\alpha}$ or the Takagi functions

$$
\left(T_{\alpha}(x)=\sum_{i=1}^{\infty} \lambda^{-\alpha i} \psi\left(\lambda^{i} x\right),\right.
$$

where $\lambda>1, \psi(x)$ is of period 1 and equals $1-|1-2 x|$ on $[0,1])$ ?

For sums of Rademacher functions other than the geometric sum, Beyer [1] proved that if the sequence $\left\{a_{i}\right\}$ is in $l_{2}$, but not in $l_{1}$, then $\sum_{i=1}^{\infty} a_{i} R_{i}(x)$ assumes every preassigned real value on a set of Hausdorff dimension 1. This implies that its graph has Hausdorff dimension 2.

\section{REFERENCES}

[1] W. A. Beyer. Hausdorff dimension of level sets of some Rademacher series. Pacific J. Math. 12 (1962), 35-46.

[2] P. ERDös. On the smoothness properties of a family of Bernoulli convolutions. Amer. J. Math. 62 (1940), 180-186.

[3] K. J. Falconer. The Geometry of Fractal Sets (Cambridge University Press, 1985).

[4] A. M. Garsia. Arithmetic properties of Bernoulli convolutions. Trans. Amer. Math. Soc. 102 (1962), 409-432. 
[5] G. H. Hardy and J. E. LitTlewood. Some properties of fractional integrals. Math. Z. 27 $(1928), 565-606$.

[6] B. JesSen and A. Wintner. Distribution functions and the Riemann zeta function. Trans. Amer. Math. Soc. 38 (1935), 48-88.

[7] J. P. Kahane and R. Salem. Sur la convolution d'une infinité de distributions de Bernoulli. Collog. Math. 6 (1958), 193-202.

[8] R. D. Mauldin and S. C. Williams. On the Hausdorff dimension of some graphs. Trans. Amer. Math. Soc. 298 (1986), 793-803.

[9] R. Salem. Algebraic Numbers and Fourier Analysis (Heath, 1963).

[10] A. Wintn Er. On convergent Poisson convolutions. Amer. J. Math. 57 (1935), 827-838. 